TITLE:

\title{
Modification of the photoelectron angular distribution through laser- induced continuum structure
}

AUTHOR(S):

Nakajima, T; Buica, G

CITATION:

Nakajima, $T$...[et al]. Modification of the photoelectron angular distribution through laserinduced continuum structure. PHYSICAL REVIEW A 2005, 71(1): 013413.

ISSUE DATE:

2005-01

URL:

http://hdl.handle.net/2433/50418

RIGHT:

Copyright 2005 American Physical Society 
PHYSICAL REVIEW A 71, 013413 (2005)

\title{
Modification of the photoelectron angular distribution through laser-induced continuum structure
}

\author{
Takashi Nakajima ${ }^{1,2, *}$ and Gabriela Buica ${ }^{1,3}$ \\ ${ }^{1}$ Institute of Advanced Energy, Kyoto University, Gokasho, Uji, Kyoto 611-0011, Japan \\ ${ }^{2}$ Institute for Molecular Science, National Institutes of Natural Sciences, Okazaki, Aichi 444-8585, Japan \\ ${ }^{3}$ Institute for Space Sciences, Bucharest, Romania \\ (Received 1 October 2004; published 19 January 2005)
}

\begin{abstract}
We theoretically investigate how the photoelectron angular distribution is altered by the introduction of a dressing laser. The physical mechanism underlying this alteration is the so-called laser-induced continuum structure; namely, a strong dressing laser induces quantum mechanical interference, the degree of which is different for different ionization channels. Therefore the branching ratio into different ionization channels changes as a function of laser detuning, and accordingly the photoelectron angular distribution is altered. After a general argument, we present specific theoretical results for the $\mathrm{K}$ atom, which indeed exhibit significant modification of the photoelectron angular distribution.
\end{abstract}

DOI: 10.1103/PhysRevA.71.013413

\section{INTRODUCTION}

Since the first experimental demonstrations on the $\mathrm{Na}$ atom some time ago $[1,2]$, it is now well established that strong radiative coupling between a bound state and a smooth continuum through a coherent radiation field induces modification in the smooth continuum. This is the so-called laser-induced continuum structure (LICS) [3]. Lots of experimental as well as theoretical work has been reported not only on atomic systems [4-10] but also on molecular systems [11-13]. Although most of the work focuses on the variation of the photoionization yield and the photodissociation products, nonlinear optical effects have also been investigated in terms of the enhancement of third-harmonic generation [14]. A little bit different scenario emerges if one considers the spin degree of freedom of the photoelectrons, namely, spinpolarized photoelectrons. In Ref. [10], we theoretically investigated the spin polarization of photoelectrons using LICS, and specific numerical examples were presented for $\mathrm{Rb}$ and $\mathrm{Cs}$.

It should be noted that the ionization line shape of LICS typically exhibits an autoionizationlike profile. As the autoionization profile is characterized by an asymmetric parameter, the ionization profile of LICS is also characterized in terms of the asymmetric parameter because of the similarity between them. If there is more than a single ionization on dissociation channel to be coupled through LICS, it seems to be a reasonable thought that it might be possible to control the branching ratio into different ionization and dissociation channels $[7,9]$. To be more specific, let us consider the case in which an initially occupied bound state and an initially unoccupied state, say, $4 p$ and $6 p$ of the $\mathrm{K}$ atom, respectively, are coupled by two lasers whose frequencies nearly satisfy two-photon resonance. In order to simplify the argument, we neglect spin-orbit interactions for a moment. Obviously there are two continua involved in the process, $\epsilon s$ and $\epsilon d$, where $\epsilon$ denotes the continuum energy. Since photoelectrons ejected

*Email address: t-nakajima@iae.kyoto-u.ac.jp
PACS number(s): $32.80 . \mathrm{Qk}, 42.50 . \mathrm{Hz}, 32.80 . \mathrm{Rm}$

into the $s$ and $d$ continua have different angular distributions, we expect that the modification of the branching ratio through LICS may result in the modification of the photoelectron angular distribution (PAD).

In this paper, we theoretically investigate the effects of LICS on PAD with specific numerical examples for the $\mathrm{K}$ atom. Resolvent and amplitude equations are employed to describe the time-dependent dynamics of the process. Relevant dipole matrix elements are computed using quantum defect theory. Since the spin angular momentum and its projection along the quantization axis affect the PAD, spin-orbit interactions are taken into account for all bound and continuum states involved in the process. As we will show in the following sections, the influence of the dressing laser on PAD is significant. The branching ratio of ionization into the two continua, $s$ and $d$ waves, turns out to vary as a function of laser detuning, and, as we expect, the variation of PAD is most significant at the laser detunings where the branching ratio becomes a maximum or minimum.

\section{THEORY}

\section{A. Model}

The system we consider is described in Fig. 1. We assume that $\mathrm{K}$ atoms are excited by the linearly polarized nanosecond auxiliary laser to the $4 p$ state of the $\mathrm{K}$ atom prior to the interaction with the probe and dressing lasers with frequencies $\omega_{p}$ and $\omega_{d}$, respectively. Depending on the frequency of the auxiliary laser, we can selectively excite $4 p_{1 / 2}$ or $4 p_{3 / 2}$ with magnetic sublevels $m_{j}= \pm 1 / 2$, which will serve as an initial state $|0\rangle$ with energy $E_{0}$ in this work. Now, the initially populated state $|0\rangle$ and initially empty state $|1\rangle$ with energy $E_{1}$, which are $6 p_{1 / 2}$ or $6 p_{3 / 2}$, are coupled by the linearly polarized probe and dressing lasers whose frequencies nearly satisfy two-photon resonance. Since we assume that the polarization axes of both lasers are parallel, the dynamics of the $4 p_{1 / 2}-6 p_{1 / 2}\left(4 p_{3 / 2}-6 p_{3 / 2}\right)$ system with $m_{j}=1 / 2$ and $-1 / 2$ are completely symmetric. Therefore, we consider the system behavior with $m_{j}=1 / 2$ only. The radiative lifetimes of $4 p_{1 / 2}$ 

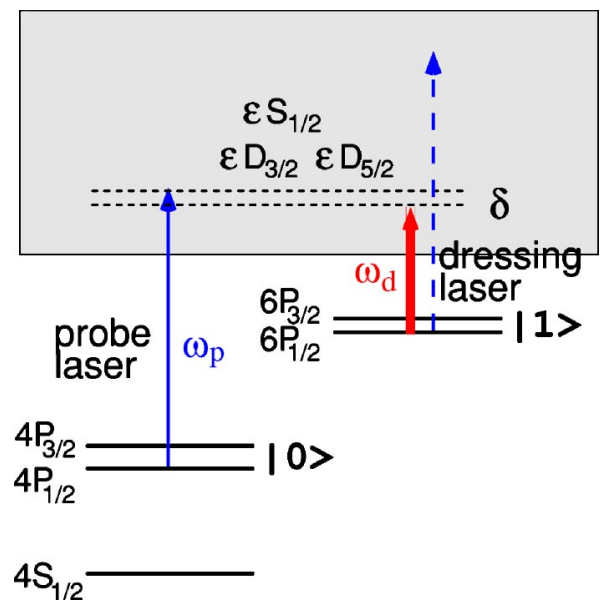

FIG. 1. Level scheme. $4 p$ and $6 p$ states of $\mathrm{K}$ atom are coupled by the linearly polarized probe and dressing lasers. With the laser intensities and the detuning range we have chosen for the present study, it is possible to consider each set of the fine-structureresolved system, the $\mathrm{K} 4 p_{1 / 2}-6 p_{1 / 2}$ system and the $\mathrm{K} 4 p_{3 / 2}-6 p_{3 / 2}$ system. Incoherent ionization from state $|1\rangle$ by the probe laser is also shown by the dashed line.

and $4 p_{3 / 2}$, and $6 p_{1 / 2}$ and $6 p_{3 / 2}$, are about 26 and $345 \mathrm{~ns}$, respectively, and will be phenomenologically included in the following analysis.

For simplicity, we further assume that the systems $4 p_{1 / 2}-6 p_{1 / 2}$ and $4 p_{3 / 2}-6 p_{3 / 2}$ are isolated from each other. Whether we may treat each system separately or not depends on the pulse durations and intensities of the lasers: If the laser intensities are sufficiently strong to induce a broad Rabi frequency to cover the fine structure of $4 p$ and $6 p$, or if the ac Stark shifts are so large as to cause shifts as large as the fine structure splitting, we have to treat all $4 p_{1 / 2}, 4 p_{3 / 2}, 6 p_{1 / 2}$, and $6 p_{3 / 2}$ states on equal footing. Furthermore, if the pulse duration is short enough to overlap the fine structure of $4 p$ or $6 p$, we have to treat all four states on an equal footing as well. However, as long as we use nanosecond lasers with appropriate intensities and detunings, it is perfectly valid to treat each $4 p_{1 / 2}-6 p_{1 / 2}$ and $4 p_{3 / 2}-6 p_{3 / 2}$ system separately, which will greatly simplify the analysis. As we will justify in the beginning of Sec. III it is indeed this intensity and detuning regime we focus on in this paper.

\section{B. Time-dependent amplitude equations}

In order to describe all the dynamics involved in Fig. 1 in a time-dependent manner, we employ a resolvent operator formalism. Now, we start with the resolvent equation $[15,16]$,

$$
\left(z-H_{0}-D^{(p)}-D^{(d)}\right) G=1,
$$

where $H_{0}$ is a field-free atomic Hamiltonian, and $D^{(p)}$ and $D^{(d)}$ are the dipole operators for the probe and dressing fields, respectively. Following the standard procedure $[15,16]$, a set of resolvent equations is obtained as follows:

$$
\begin{gathered}
{\left[z-E_{0}-\hbar \omega_{p}-\left(S_{0}^{(p)}+S_{0}^{(d)}\right)+i \frac{\Gamma_{0}^{(p)}}{2}\right] G_{0}-\Omega\left(1-\frac{i}{q}\right) G_{1}=1} \\
-\Omega\left(1-\frac{i}{q}\right) G_{0}+\left[z-E_{1}-\hbar \omega_{d}-\left(S_{1}^{(p)}+S_{1}^{(d)}\right)\right. \\
\left.+\frac{i}{2}\left(\Gamma_{1}^{(p)}+\Gamma_{1}^{(d)}\right)\right] G_{1}=0 .
\end{gathered}
$$

$\Gamma_{j}^{(\alpha)}$, s are the ionization widths of state $|j\rangle(j=0$ or 1$)$ by the probe $(\alpha=p)$ or dressing laser $(\alpha=d)$ into all the possible continua. In particular, $\Gamma_{1}^{(p)}$ is an incoherent ionization width from state $|1\rangle$ by the probe laser, as described by the dashed line in Fig. 1. $\Omega$ is an effective two-photon Rabi frequency between $|0\rangle$ and $|1\rangle$, and $q$ is an asymmetry parameter. The $S_{j}^{(\alpha)}$ 's are the ac Stark shifts of state $|j\rangle$ by laser $p$ or $d$. Note that all the continuum states $|c\rangle$ have already been eliminated in Eqs. (2) and (3). In the above equations the two-photon Rabi frequency $\Omega$ is a sum of the two-photon Rabi frequencies through the $\epsilon s$ and $\epsilon d$ continua, i.e.,

$$
\Omega\left(1-\frac{i}{q}\right)=\sum_{\beta=c_{1}, c_{2}} \Omega_{\beta}\left(1-\frac{i}{q_{\beta}}\right) .
$$

As for the equation for the continuum associated with coherent ionization, it reads

$$
-D_{c 0}^{(p)} G_{0}-D_{c 1}^{(d)} G_{1}+\left(z-E_{c}\right) G_{c}^{c o h}=0,
$$

where $D_{c j}^{(\alpha)}(j=0,1$ and $\alpha=p, d)$ are the bound-free matrix elements from state $|j\rangle$ by the probe or dressing laser. The superscript of $G_{c}^{c o h}$ implies that it is a resolvent matrix associated with the coherent continuum. To avoid complication, we have phenomenologically included incoherent ionization as $\Gamma_{1}^{(p)}$ in Eq. (3).

For the time-varying laser intensities, we have to convert the resolvent equations, Eqs. (2), (3), and (5), into amplitude equations. They read

$$
\begin{gathered}
\dot{u}_{0}=-\frac{1}{2}\left(\gamma_{0}+\Gamma_{0}^{(p)}\right) u_{0}-i \Omega\left(1-\frac{i}{q}\right) u_{1}, \\
\dot{u}_{1}=\left[i\left(\delta_{\text {static }}+\delta_{\text {Stark }}\right)-\frac{1}{2}\left(\gamma_{1}+\Gamma_{1}^{(p)}+\Gamma_{1}^{(d)}\right)\right] u_{1} \\
-i \Omega\left(1-\frac{i}{q}\right) u_{0}, \\
\dot{u}_{c}^{c o h}=-i \delta_{c} u_{c}^{c o h}-i D_{c 0}^{(p)} u_{0}-i D_{c 1}^{(d)} u_{1},
\end{gathered}
$$

where the $u_{j}$ 's $(j=0,1, c)$ are the probability amplitudes of states $|j\rangle$. The superscript of $u_{c}$ implies that it is a coherent continuum. $\delta_{\text {static }}$ is the static two-photon detuning defined by $\delta_{\text {static }}=\left(E_{0}+\hbar \omega_{p}\right)-\left(E_{1}+\hbar \omega_{d}\right)$, and $\delta_{\text {Stark }}$ is the total dynamic ac Stark shift defined as $\delta_{\text {Stark }}=\left(S_{0}^{(p)}+S_{0}^{(d)}\right)-\left(S_{1}^{(p)}\right.$ $\left.+S_{1}^{(d)}\right)$. $D_{j k}^{(\alpha)}$ 's are the bound-free matrix elements by laser $\alpha$ $(\alpha=p$ or $d$ ) from the bound state $|j\rangle$ to the continuum $|c\rangle$, which are connected to the partial ionization widths by the 
relation $\Gamma_{j k}^{(\alpha)}=2 \pi\left|D_{j k}^{(\alpha)}\right|^{2} \cdot \gamma_{j}(j=0,1)$ are the phenomenologically introduced spontaneous decay rates of states $|j\rangle$. In the following, we introduce the total detuning $\delta\left(=\delta_{\text {static }}+\delta_{\text {Stark }}\right)$, and the total decay rates of states $|0\rangle$ and $|1\rangle$ are rewritten to be $\widetilde{\Gamma}_{0}$ and $\widetilde{\Gamma}_{1}$, i.e., $\widetilde{\Gamma}_{0} \equiv \gamma_{0}+\Gamma_{0}^{(p)}$ and $\widetilde{\Gamma}_{1} \equiv \gamma_{1}+\Gamma_{1}^{(p)}+\Gamma_{1}^{(d)}$.

For the calculation of ionization yield into each continuum, it turns out that the following density matrix equations associated with the coherent continuum are useful:

$$
\begin{gathered}
\dot{\rho}_{c c}^{c o h}=2 D_{c 0}^{(p)} \operatorname{Im} \rho_{0 c}^{c o h}+2 D_{c 1}^{(d)} \operatorname{Im} \rho_{1 c}^{c o h}, \\
\dot{\rho}_{0 c}^{c o h}=\left(i \delta_{c}-\frac{1}{2} \widetilde{\Gamma}_{0}\right) \rho_{0 c}^{c o h}+i D_{c 0}^{(p) *}\left|u_{0}\right|^{2}+i D_{c 1}^{(d) *} u_{0} u_{1}^{*}, \\
\dot{\rho}_{1 c}^{c o h}=\left[i\left(\delta-\delta_{c}\right)-\frac{1}{2} \widetilde{\Gamma}_{1}\right] \rho_{1 c}^{c o h}+i D_{c 0}^{(p) *} u_{1} u_{0}^{*}+i D_{c 1}^{(d) *}\left|u_{1}\right|^{2} .
\end{gathered}
$$

By setting the time derivatives of Eqs. (10) and (11) to be zero and adiabatically eliminating $\rho_{0 c}^{c o h}$ and $\rho_{1 c}^{c o h}$ in Eq. (9), we find

$$
\begin{aligned}
\dot{\rho}_{c c}^{c o h}= & \widetilde{\Gamma}_{0} \frac{\left|D_{0 c}^{(p)}\right|^{2}\left|u_{0}\right|^{2}+D_{c 1}^{(d) *} D_{c 0} u_{0} u_{1}^{*}}{\delta_{c}^{2}+\left(\widetilde{\Gamma}_{0} / 2\right)^{2}} \\
& +\widetilde{\Gamma}_{1} \frac{D_{c 0}^{(p) *} D_{c 1}^{(d)} u_{0}^{*} u_{1}+\left|D_{c 1}^{(d)}\right|^{2}\left|u_{1}\right|^{2}}{\left(\delta-\delta_{c}\right)^{2}+\left(\widetilde{\Gamma}_{1} / 2\right)^{2}} .
\end{aligned}
$$

Furthermore, because we may safely assume that $D_{0 c}^{(p)}$ and $D_{c 1}^{(d)}$ are approximately constant over all $\delta_{c}$, they may be pulled out of the integral when we perform integration over $\delta_{c}$. Therefore, defining the ionization yield through the coherent channel as $R_{c}^{c o h}$, we obtain the expression for its time derivative to be

$$
\begin{aligned}
\frac{d R_{c}^{c o h}}{d t}= & \int d \delta_{c} \dot{\rho}_{c c}^{c c h} \\
= & \widetilde{\Gamma}_{0}\left(\left|D_{0 c}^{(p)}\right|^{2}\left|u_{0}\right|^{2}+D_{c 1}^{(d) *} D_{c 0}^{(p)} u_{0} u_{1}\right) \int d \delta_{c} \frac{1}{\delta_{c}^{2}+\left(\widetilde{\Gamma}_{0} / 2\right)^{2}} \\
& +\widetilde{\Gamma}_{1}\left(\left|D_{c 1}^{(d)}\right|^{2}\left|u_{1}\right|^{2}+D_{c 0}^{(p) *} D_{c 1}^{(d)} u_{0}^{*} u_{1}\right) \\
& \times \int d \delta_{c} \frac{1}{\left(\delta-\delta_{c}\right)^{2}+\left(\widetilde{\Gamma}_{1} / 2\right)^{2}}=2 \pi\left|D_{0 c}^{(p)} u_{0}+D_{c 1}^{(d)} u_{1}\right|^{2} .
\end{aligned}
$$

Hence

$$
R_{c}^{c o h}=2 \pi \int d t\left|D_{0 c}^{(p)} u_{0}+D_{c 1}^{(d)} u_{1}\right|^{2},
$$

which is equivalent to Eqs. (9) and (10) in Ref. [10]. If there are two coherent continua, extension of the above procedure to the case of two continua is rather straightforward, and the result is written as

$$
R^{c o h}=2 \pi \int d t \sum_{c=c_{1}, c_{2}}\left|D_{0 c}^{(p)} u_{0}+D_{c 1}^{(d)} u_{1}\right|^{2} .
$$

Including incoherent ionization, we finally obtain the total ionization yield $R$ as

$$
\begin{aligned}
R & =R^{c o h}+R^{i n c} \\
& =2 \pi \int d t\left[\sum_{c=c_{1}, c_{2}}\left|D_{0 c}^{(p)} u_{0}+D_{c 1}^{(d)} u_{1}\right|^{2}+\sum_{c=c_{3}, c_{4}}\left|D_{c 1}^{(p)} u_{1}\right|^{2}\right] .
\end{aligned}
$$

Given the laser intensity we have chosen for the numerical results, however, the contribution of incoherent ionization by the probe laser turns out to be negligibly small.

\section{Photoelectron angular distribution}

To derive all equations in the previous subsection, we have implicitly assumed that the angle integration has already been performed for the bound-free dipole moments. For the purpose of calculating photoelectron angular distribution, however, we need equations before angle integration. Accordingly, it is necessary to examine Eqs. (8)-(11) more carefully. Using a partial wave expansion, the continuum of alkali-metal atoms can be represented by

$$
\begin{aligned}
\left|\vec{k} ; m_{s}\right\rangle= & \sum_{l, m_{l}} a_{l m_{l}}\left|\vec{k} ; l m_{l}\right\rangle\left|1 / 2 m_{s}\right\rangle \\
= & \sum_{l, m_{l}, j} a_{l m_{l}}(-1)^{l-1 / 2+m_{l}+m_{s}} \sqrt{2 j+1} \\
& \times\left(\begin{array}{ccc}
l & 1 / 2 & j \\
m_{l} & m_{s} & -\left(m_{l}+m_{s}\right)
\end{array}\right)\left|\vec{k} ;(l s) j m_{j}\right\rangle,
\end{aligned}
$$

where $\vec{k}$ is the wave vector of the photoelectron, $a_{l m_{l}}$ $=4 \pi i^{l} e^{-i \delta_{l} Y_{l m_{l}}}(\Theta, \Phi)$, and $\delta_{l}$ is the phase shift, which is a sum of the Coulomb phase shift and the scattering phase shift. For our specific case (see Fig. 1), the partial waves $\epsilon S$ and $\epsilon d$ are of importance since they contribute to the boundfree matrix elements from states $|0\rangle$ and $|1\rangle$. After the angle integration, those two continua can be treated separately since they are orthogonal to each other, as we have already implied in Eq. (15). However, before angle integration, they interfere differently at different angles, and cannot be treated separately. Therefore, the correct expression of the photoelectron yield before angle integration is

$$
\begin{aligned}
\frac{d R}{d \Omega}= & 2 \pi \int d t\left[\left|\sum_{c=\epsilon s, \epsilon d}\left[D_{0 c}^{(p)}(\Theta, \Phi) u_{0}+D_{c 1}^{(d)}(\Theta, \Phi) u_{1}\right]\right|^{2}\right. \\
& \left.+\left|\sum_{c=\epsilon s, \epsilon d} D_{c 1}^{(p)}(\Theta, \Phi) u_{1}\right|^{2}\right]
\end{aligned}
$$

where the second term in Eq. (18) represents the contribution due to the incoherent ionization channel. Clearly, when the angle integration is performed for $d R / d \Omega$, some of the cross terms in Eq. (18) vanish due to the orthogonality of the spherical harmonics, and Eq. (18) is reduced to Eq. (16). Having understood the above argument, it is easy to write down the following formula for the PAD: 


$$
\begin{aligned}
\frac{d R}{d \Omega}= & 2 \pi \int d t \sum_{m_{s}= \pm 1 / 2}\left[\left|\left\langle\vec{k} ; m_{s}\left|D^{(p)}\right| 0\right\rangle u_{0}+\left\langle\vec{k} ; m_{s}\left|D^{(d)}\right| 1\right\rangle u_{1}\right|^{2}\right. \\
& \left.+\left|\left\langle\vec{k} ; m_{s}\left|D^{(p)}\right| 1\right\rangle u_{1}\right|^{2}\right] .
\end{aligned}
$$

Finally we obtain the general expression of the PAD, applicable for both $4 p_{1 / 2}-6 p_{1 / 2}$ and $4 p_{3 / 2}-6 p_{3 / 2}$ systems, as

$$
\begin{aligned}
\frac{d R}{d t d \Omega}= & 0.589 \pi \sum_{m_{s}= \pm 1 / 2}\left[\mid \sqrt{\Gamma_{0}^{\left(p, m_{s}\right)}(\Theta, \Phi)} u_{0}\right. \\
& \left.+\left.\sqrt{\Gamma_{1}^{\left(d, m_{s}\right)}(\Theta, \Phi)} u_{1}\right|^{2}+\left|\sqrt{\Gamma_{1}^{\left(p, m_{s}\right)}(\Theta, \Phi)} u_{1}\right|^{2}\right],
\end{aligned}
$$

with $0.589 \pi$ being a conversion factor for the appropriate normalization; we have introduced the differential ionization widths defined as

$$
\begin{aligned}
\Gamma_{j}^{(\alpha,+1 / 2)}(\Theta, \Phi)= & -\frac{1}{3} R_{j \epsilon s}^{(\alpha)} e^{i \delta_{s}} Y_{00}(\Theta, \Phi) \\
& +\left.\frac{2}{3 \sqrt{5}} R_{j \epsilon d}^{(\alpha)} e^{i \delta_{d}} Y_{20}(\Theta, \Phi)\right|^{2} I_{\alpha}, \\
\Gamma_{j}^{(\alpha,-1 / 2)}(\Theta, \Phi)=\mid & -\sqrt{\frac{2}{15}} R_{j \in d}^{(\alpha)} e^{i \delta_{d}} Y_{21}(\Theta, \Phi)||^{2} I_{\alpha} .
\end{aligned}
$$

In Eqs. (21) and (22), $R_{j \in s}^{(\alpha)}(j=4 p, 6 p)$, etc., represents a radial bound-free matrix element from state $j$ to the continuum $\epsilon s$ by laser $\alpha(\alpha=p$ or $d)$ and evaluated in atomic units, and the laser intensities $I_{p}$ and $I_{d}$ are in $\mathrm{W} / \mathrm{cm}^{2}$. For the coherent continuum, the relevant phase shifts are $\delta_{s}=1.937$ and $\delta_{d}=$ -6.574 , which are the sums of the Coulomb phase shifts $\delta_{s}^{C}=-4.924$ and $\delta_{d}^{C}=-7.551$ and the scattering phase shifts $\pi \mu_{s}=6.861$ and $\pi \mu_{d}=0.977$ with $\mu_{l}(l=s, d)$ being the quantum defects estimated from the linear extrapolation of the bound Rydberg $s$ and $d$ series of the $\mathrm{K}$ atom to the continuum energy of interest. Similar evaluation of phase shifts has also been carried out for the incoherent continuum. Equation (20) together with Eqs. (21) and (22) gives the PAD for the $4 p_{1 / 2}-6 p_{1 / 2}$ system with appropriate normalization such that the angle-integrated quantity becomes identical to the total ionization yield calculated with Eq. (16). Equation (20) is also applicable for the PAD of the $4 p_{3 / 2}-6 p_{3 / 2}$ system only if the differential ionization widths are employed, which read

$$
\begin{aligned}
\Gamma_{j}^{(\alpha,+1 / 2)}(\Theta, \Phi)= & \mid \frac{\sqrt{2}}{3} R_{j \epsilon \epsilon}^{(\alpha)} e^{i \delta_{s}} Y_{00}(\Theta, \Phi) \\
& -\left.\frac{2 \sqrt{10}}{15} R_{j \epsilon d}^{(\alpha)} e^{i \delta_{d}} Y_{20}(\Theta, \Phi)\right|^{2} I_{\alpha}, \\
\Gamma_{j}^{(\alpha,-1 / 2)}(\Theta, \Phi)= & \left|-\frac{1}{\sqrt{15}} R_{j \epsilon d}^{(\alpha)} e^{i \delta_{d}} Y_{21}(\Theta, \Phi)\right|^{2} I_{\alpha} .
\end{aligned}
$$

\section{NUMERICAL RESULTS AND DISCUSSION}

In order to solve the set of time-dependent amplitude equations Eqs. (6) and (7), we need atomic parameters such
TABLE I. Atomic parameters for the $\mathrm{K} 4 P_{1 / 2}-6 p_{1 / 2}$ system. $\Omega$ in $\mathrm{rad} / \mathrm{s}, \Gamma$ in s$~^{-1}, S$ in rad/s, and $I_{p}$ and $I_{d}$ in $\mathrm{W} / \mathrm{cm}^{2}$.

\begin{tabular}{cccc}
\hline \hline & & & \\
$\Omega$ & $-8.12 \sqrt{I_{p} I_{d}}$ & $q$ & -0.91 \\
$\Omega_{\epsilon s}$ & $3.47 \sqrt{I_{p} I_{d}}$ & $q_{\epsilon s}$ & 1.71 \\
$\Omega_{\epsilon d}$ & $-11.58 \sqrt{I_{p} I_{d}}$ & $q_{\epsilon d}$ & -1.69 \\
$\Gamma_{0}^{(p)}$ & $11.59 I_{p}$ & $S_{0}^{(p)}$ & $14.1 I_{p}$ \\
$\Gamma_{1}^{(d)}$ & $28.04 I_{d}$ & $S_{0}^{(d)}$ & $947.5 I_{d}$ \\
$\Gamma_{1}^{(p)}$ & $3.66 I_{p}$ & $S_{1}^{(p)}$ & $21.04 I_{p}$ \\
& & $S_{1}^{(d)}$ & $86.9 I_{d}$ \\
\hline \hline
\end{tabular}

as the Rabi frequency $\Omega$, the asymmetry parameter $q$, the ionization widths $\Gamma$, and the ac Stark shifts $S$. All the necessary single- and effective two-photon dipole matrix elements are obtained using quantum defect theory and the Green function technique. Calculated atomic parameters are listed in Tables I and II for the $\mathrm{K} 4 p_{1 / 2}-6 p_{1 / 2}$ and $4 p_{3 / 2}-6 p_{3 / 2}$ systems, respectively. By substituting those parameters into Eqs. (6) and (7), we can easily solve those equations for the given peak intensities, detunings, and the temporal profile of the lasers. In this work we have assumed that the temporal profile of the probe and dressing lasers is Gaussian and delayed from the auxiliary laser by $30 \mathrm{~ns}$ so that the influence of the auxiliary laser, other than the pumping of state $|0\rangle$, does not have to be taken into account. In all the numerical results presented in this section, the zero point of the total detuning, $\delta=0$, has been chosen at the peak intensity so that $\delta_{\text {static }}$ $+\delta_{\text {Stark }}^{\text {max }}=0$; namely, $\delta=0$ means that $\delta_{\text {static }}=-\delta_{\text {Stark }}^{\text {max }}$. Once the solution is obtained for $u_{0}(t)$ and $u_{1}(t)$, the partial as well as total ionization yield into each continuum can be calculated from Eqs. (14) and (16). In the following subsections, we present results for the $\mathrm{K} 4 p_{1 / 2}-6 p_{1 / 2}$ and $4 p_{3 / 2}-6 p_{3 / 2}$ systems.

Now, as promised in Sec. II, it is time to justify why the two systems $\mathrm{K} 4 p_{1 / 2}-6 p_{1 / 2}$ and $4 p_{3 / 2}-6 p_{3 / 2}$, may be treated separately. As one sees from Tables I and II, the largest contribution of the ac Stark shift comes from the dressing laser for state $|0\rangle$. Furthermore, both states $|0\rangle$ and $|1\rangle$ shift to the same direction, and what matters is the relative shift between the $\mathrm{K} 4 p_{1 / 2}-6 p_{1 / 2}$ and $4 p_{3 / 2}-6 p_{3 / 2}$ systems, which is about $280 I_{d}(\mathrm{rad} / \mathrm{s})$. Given the maximum intensity of the dressing laser considered in this work, $I_{d}=500 \mathrm{MW} / \mathrm{cm}^{2}$, the relative shift of both systems is $280 I_{d}=1.4 \times 10^{11}(\mathrm{rad} / \mathrm{s})$, which is less than $1 \mathrm{~cm}^{-1}$. Knowing the fact that the fine structure energy splittings of $4 p$ and $6 p$ are 58 and $8 \mathrm{~cm}^{-1}$, respec-

TABLE II. Atomic parameters for the $\mathrm{K} 4 P_{3 / 2}-6 p_{3 / 2}$ system. $\Omega$ in $\mathrm{rad} / \mathrm{s}, \Gamma$ in s${ }^{-1}, S$ in rad/s, and $I_{p}$ and $I_{d}$ in $\mathrm{W} / \mathrm{cm}^{2}$.

\begin{tabular}{cccr}
\hline \hline & & & \\
$\Omega$ & $-5.80 \sqrt{I_{p} I_{d}}$ & $q$ & -0.5 \\
$\Omega_{\epsilon s}$ & $6.94 \sqrt{I_{p} I_{d}}$ & $q_{\epsilon s}$ & 1.71 \\
$\Omega_{\epsilon d}$ & $-12.74 \sqrt{I_{p} I_{d}}$ & $q_{\epsilon d}$ & -1.69 \\
$\Gamma_{0}^{(p)}$ & $14.46 I_{p}$ & $S_{0}^{(p)}$ & $12.3 I_{p}$ \\
$\Gamma_{1}^{(d)}$ & $38.57 I_{d}$ & $S_{0}^{(d)}$ & $1231.8 I_{d}$ \\
$\Gamma_{1}^{(p)}$ & $4.32 I_{p}$ & $S_{1}^{(p)}$ & $20.8 I_{p}$ \\
& & $S_{1}^{(d)}$ & $97.0 I_{d}$ \\
\hline \hline
\end{tabular}




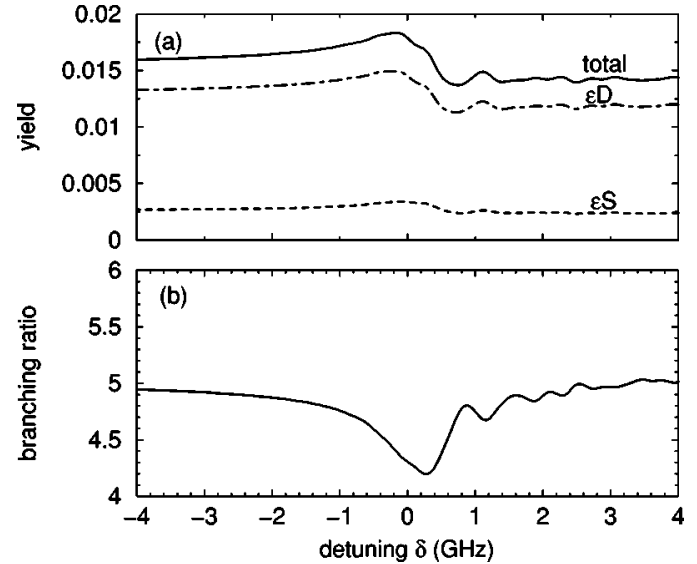

FIG. 2. (a) Total and partial ionization yields into each $\epsilon s$ and $\epsilon d$ continuum, and (b) the branching ratio between them for the $\mathrm{K}$ $4 p_{1 / 2}-6 p_{1 / 2}$ system as a function of two-photon detuning $\delta$. Pulse durations and peak laser intensities are chosen to be $\tau_{p}=4 \mathrm{~ns}$ and $I_{p}=1 \mathrm{MW} / \mathrm{cm}^{2}$, and $\tau_{d}=4 \mathrm{~ns}$ and $I_{d}=100 \mathrm{MW} / \mathrm{cm}^{2}$, for the probe and dressing lasers, respectively.

tively, the ac Stark shifts are still much smaller than the energy splittings. Since the LICS structure we see in the following subsections appears in the detuning range of a few gigahertz, which is less than $1 \mathrm{~cm}^{-1}$, it is clear that we may treat each system separately.

\section{A. K $4 p_{1 / 2}-6 p_{1 / 2}$ system}

One of the major obstacles to observing clear LICS is the ac Stark shift, since it causes time-dependent dynamic detuning during the pulse. From Table I it can be seen that $S_{0}^{(d)}$ is significantly large. For $I_{d}=100 \mathrm{MW} / \mathrm{cm}^{2}$, it is as large as $0.5 \mathrm{GHz}$ and much larger than the Rabi frequency and ionization widths. If the pulse durations of the probe and dressing lasers are comparable, the subtle structure of LICS as a function of total detuning may be easily smeared out. Indeed, for the case of probe pulse duration $\tau_{p}=4 \mathrm{~ns}$ [full width at half maximum (FWHM)] and the dressing laser pulse duration $\tau_{d}=4 \mathrm{~ns}(\mathrm{FWHM})$ with the peak intensities of $I_{p}=1$ and

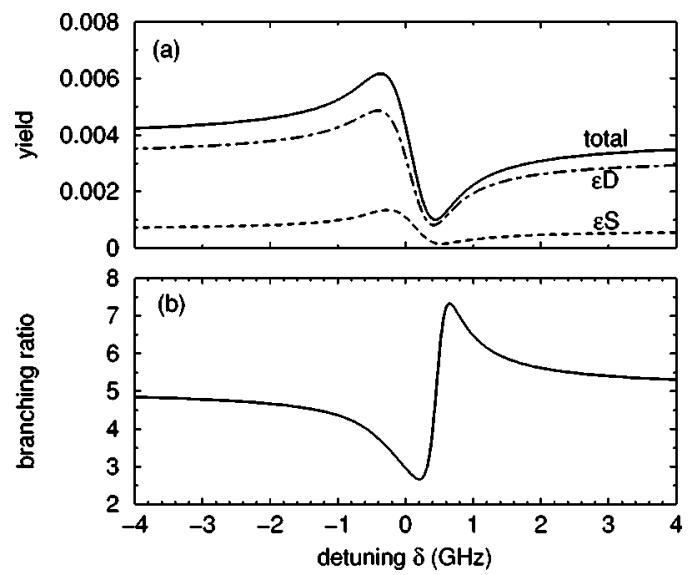

FIG. 3. Same as Fig. 2 only with the different pulse durations of $\tau_{p}=1 \mathrm{~ns}$ and $\tau_{d}=10 \mathrm{~ns}$ for the probe and dressing lasers, respectively.
$I_{d}=100 \mathrm{MW} / \mathrm{cm}^{2}$, we do not see so much structure, as shown in Fig. 2. To circumvent this problem, we have changed the pulse durations to be $\tau_{p}=1 \mathrm{~ns}$ (FWHM) and $\tau_{d}$ $=10 \mathrm{~ns}$ (FWHM) with the same peak intensities. The result is shown in Fig. 3(a). As expected, the LICS profile becomes more eminent. For the purpose of plotting PAD, the branching ratio, which is defined as a ratio of the yield into $d$ continuum divided by that into the $s$ continuum, is also plotted in Fig. 3(b). Since PAD is very different for the $s$ and $d$ electrons, the variation of the branching ratio is a good indicator for the variation of PAD. The three-dimensional PAD is shown in Figs. 4(a)-4(c) for the three specific cases, corresponding to the branching ratios at far off resonance $(\delta=$ $-4 \mathrm{GHz})$, at the minimum $(\delta=0.2 \mathrm{GHz})$, and at the maximum $(\delta=0.64 \mathrm{GHz})$. It is clear that the PADs are significantly different at different detunings.

Frequently PAD is represented as a superposition of $\cos ^{2 n} \theta$ functions, namely,

$$
\frac{d R}{d \Omega}=\sum_{n=0,1,2, \ldots} \beta_{2 n} \cos ^{2 n} \theta,
$$

where $\beta_{2 n}$ is called the asymmetry parameter and gives the deviation of PAD from the spherically symmetric distribution which corresponds to $\beta_{2 n}=0$. For the case of the $4 p_{1 / 2}-6 p_{1 / 2}$ system, the asymmetry parameters are nonzero only up to $n=1$. It would be instructive to plot the asymmetry parameters as a function of detuning. For that purpose, we rewrite the spherical harmonics in Eq. (20) in terms of the $\cos ^{2 n} \theta$ functions. After some straightforward algebra, we obtain

$$
\begin{gathered}
\beta_{0}=0.589 \int d t\left[\left|\frac{1}{2} A-\frac{\sqrt{5}}{4} B\right|^{2}+\left|\frac{1}{2} C-\frac{\sqrt{5}}{4} D\right|^{2}\right], \\
\beta_{2}=0.589 \int d t\left[\frac{3 \sqrt{5}}{4} \operatorname{Re}(A B *)+\frac{15}{16}|B|^{2}\right. \\
\left.+\frac{3 \sqrt{5}}{4} \operatorname{Re}(C D *)+\frac{15}{16}|D|^{2}\right]
\end{gathered}
$$

where

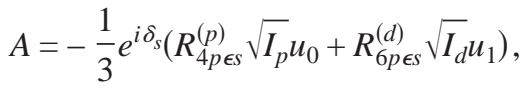

$$
\begin{aligned}
& B=\frac{2}{3 \sqrt{5}} e^{i \delta_{d}}\left(R_{4 p \epsilon d}^{(p)} \sqrt{I_{p}} u_{0}+R_{6 p \epsilon d}^{(d)} \sqrt{I_{d}} u_{1}\right), \\
& C=-\frac{1}{3} e^{i \delta_{s}} R_{6 p \epsilon s}^{(p)} \sqrt{I_{p}} u_{1}, \\
& D=\frac{2}{3 \sqrt{5}} e^{i \delta_{d}} R_{6 p \epsilon d}^{(p)} \sqrt{I_{p}} u_{1} .
\end{aligned}
$$

Figure 5 shows the variation of $\beta_{0}$ and $\beta_{2} / \beta_{0}$ as a function of detuning $\delta$ at three different dressing laser intensities 


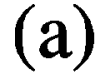

(b)

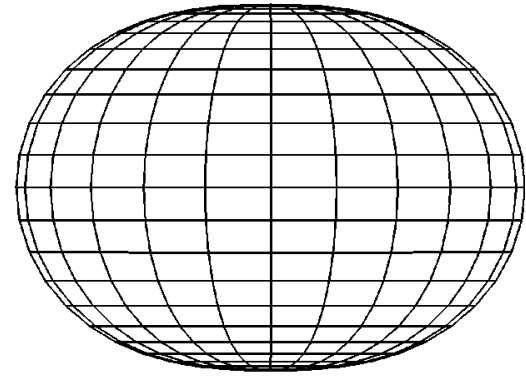

(c)

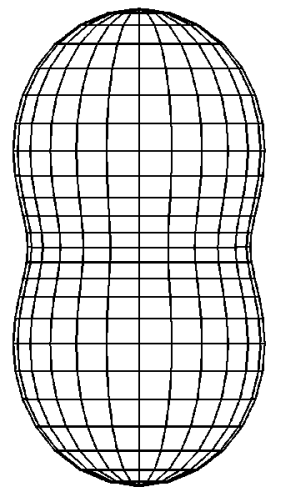

FIG. 4. Three-dimensional photoelectron angular distribution for the $\mathrm{K} 4 p_{1 / 2}-6 p_{1 / 2}$ system at three different two-photon detunings $\delta=-4,0.2$, and $0.64 \mathrm{GHz}$. Pulse durations and peak intensities are $\tau_{p}=1 \mathrm{~ns}$ and $I_{p}=1 \mathrm{MW} / \mathrm{cm}^{2}$ for the probe laser, and $\tau_{d}=10 \mathrm{~ns}$ and $I_{p}=100 \mathrm{MW} / \mathrm{cm}^{2}$ for the dressing laser.

$I_{d}=100,200$, and $500 \mathrm{MW} / \mathrm{cm}^{2}$ with the probe laser intensity and the pulse duration fixed to be $I_{p}=1 \mathrm{MW} / \mathrm{cm}^{2}$ and $\tau_{p}=1 \mathrm{~ns}$ and $\tau_{d}=10 \mathrm{~ns}$. As we have already seen in Figs. 3 and 4 , the asymmetry parameters vary significantly near resonance. In addition, the higher the dressing laser intensity is, the broader the structure becomes. This is simply due to the broader ionization width for $\Gamma_{1}$ which is similar to the case of broad autoionization width. It is interesting to note that not only the ionization spectra but also the variation of $\beta_{2 n}$ also exhibit an asymmetric profile.

\section{B. K $4 p_{3 / 2}-6 p_{3 / 2}$ system}

We now turn to the case of the $4 p_{3 / 2}-6 p_{3 / 2}$ system. From Table II, it is seen that the ac Stark shift $S_{0}^{(d)}$ for the $4 p_{3 / 2}-6 p_{3 / 2}$ system is even larger than that for the $4 p_{1 / 2}$ $-6 p_{1 / 2}$ system. This is basically due to the different angular coefficients between the two systems. For a similar reason as
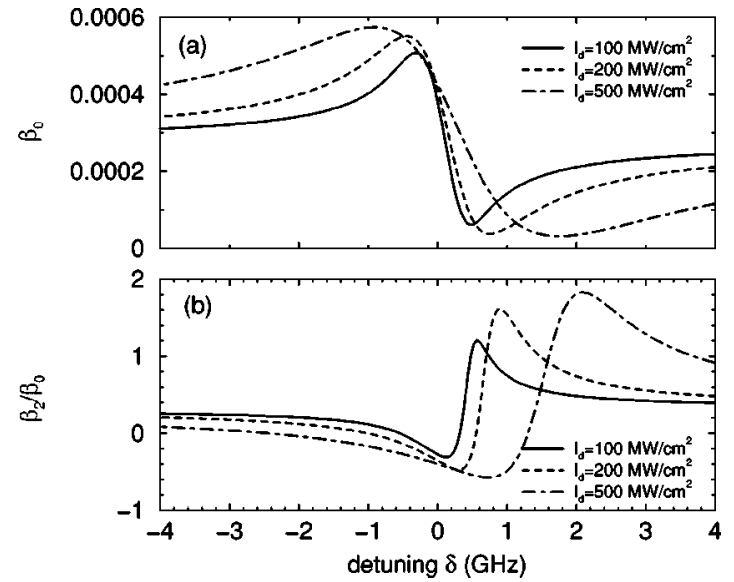

FIG. 5. Variations of the asymmetry parameters $\beta_{0}$ and $\beta_{2}$ for the $\mathrm{K} 4 p_{1 / 2}-6 p_{1 / 2}$ system as a function of two-photon detuning $\delta$ for the three different dressing laser intensities $I_{d}=100,200$, and $500 \mathrm{MW} / \mathrm{cm}^{2}$. All other parameters are kept the same as those for Fig. 3, i.e., $\tau_{p}=1 \mathrm{~ns}, \tau_{d}=10 \mathrm{~ns}$, and $I_{p}=1 \mathrm{MW} / \mathrm{cm}^{2}$.

explained in Sec. III A, it is clear, as we have already seen in Fig. 2, that the pulse duration for the dressing laser must be substantially longer than that for the probe laser to observe a nice LICS profile. Therefore, all the results we show in this subsection is for $\tau_{p}=1 \mathrm{~ns}$ and $\tau_{d}=10 \mathrm{~ns}$.

Figure 6 shows the variation of the total as well as partial ionization yield as a function of detuning. The structure in Fig. 6(a) is similar to that in Fig. 3(a), but not quite. This is due to the fact that, although $q_{\epsilon s}$ and $q_{\epsilon d}$ are the same for both systems as one obviously sees from Tables I and II, the $q$ itself is different because of the different $\Omega$ 's in both systems. This can be easily checked using Eq. (4). More interestingly, the variation of the branching ratio shown in Fig. 6(b) is substantially smaller than that shown in Fig. 3(b). Therefore, we intuitively expect that the modification of PAD might be smaller for this system. In order to examine our speculation, we plot the three-dimensional PAD in Fig. 7 for

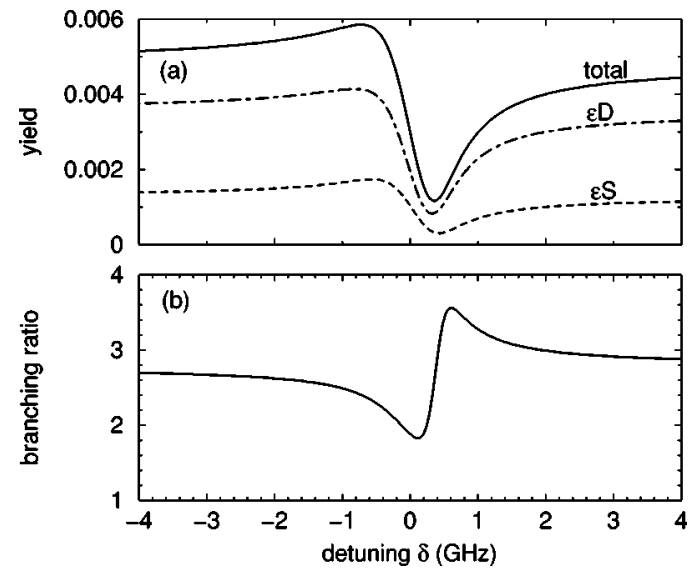

FIG. 6. (a) Total and partial ionization yields into each $\epsilon s$ and $\epsilon d$ continuum, and (b) the branching ratio between them for the $\mathrm{K}$ $4 p_{3 / 2}-6 p_{3 / 2}$ system as a function of two-photon detuning $\delta$. Pulse durations and peak laser intensities are $\tau_{p}=1 \mathrm{~ns}$ and $I_{p}$ $=1 \mathrm{MW} / \mathrm{cm}^{2}$, and $\tau_{d}=10 \mathrm{~ns}$ and $I_{d}=100 \mathrm{MW} / \mathrm{cm}^{2}$, for the probe and dressing lasers, respectively. 
(a)

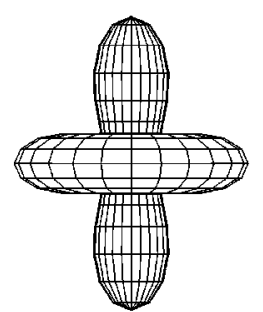

(b)

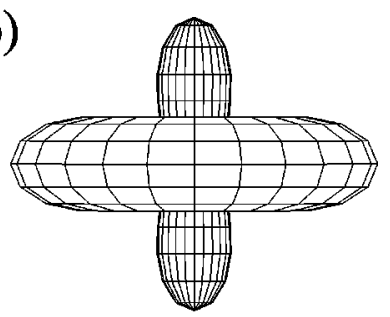

(c)

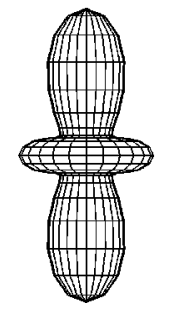

FIG. 7. Three-dimensional photoelectron angular distribution for the $\mathrm{K} 4 p_{3 / 2}-6 p_{3 / 2}$ system at three different two-photon detunings $\delta=-4,0.12$, and $0.6 \mathrm{GHz}$. Pulse durations and peak intensities are $\tau_{p}=1 \mathrm{~ns}$ and $I_{p}=1 \mathrm{MW} / \mathrm{cm}^{2}$ for the probe laser, and $\tau_{d}=10 \mathrm{~ns}$ and $I_{p}=100 \mathrm{MW} / \mathrm{cm}^{2}$ for the dressing laser.

the three specific detunings, corresponding to the branching ratios at far off resonance, $\delta=-4 \mathrm{GHz}$, at the minimum, $\delta$ $=0.12 \mathrm{GHz}$, and at the maximum, $\delta=0.6 \mathrm{GHz}$. The modification of the 3D PAD is more than we expect: Variation of the sidelobe, which is absent for the $4 p_{1 / 2}-6 p_{1 / 2}$ system because $\beta_{4}=0$, is striking.

The asymmetry parameters $\beta_{2 n}$ are now nonzero up to $n$ $=2$. After some algebra, we obtain

$$
\begin{gathered}
\beta_{0}=0.589 \int d t\left[\left|\frac{1}{2} A-\frac{\sqrt{5}}{4} B\right|^{2}+\left|\frac{1}{2} C-\frac{\sqrt{5}}{4} D\right|^{2}\right], \\
\beta_{2}=0.589 \int d t\left[\frac{3 \sqrt{5}}{4} \operatorname{Re}(A B *)-\frac{75}{64}|B|^{2}\right. \\
\left.+\frac{3 \sqrt{5}}{4} \operatorname{Re}(C D *)-\frac{75}{64}|D|^{2}\right], \\
\beta_{4}=0.589 \int d t \frac{135}{64}\left[|B|^{2}+|D|^{2}\right],
\end{gathered}
$$

where

$$
A=\frac{\sqrt{2}}{3} e^{i \delta_{s}}\left(R_{4 p \epsilon s}^{(p)} \sqrt{I_{p}} u_{0}+R_{6 p \epsilon s}^{(d)} \sqrt{I_{d}} u_{1}\right)
$$

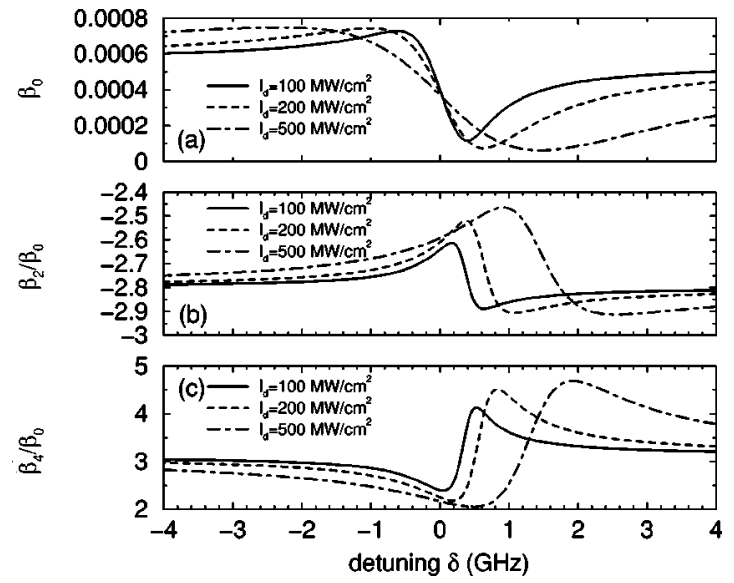

FIG. 8. Variations of the asymmetry parameters $\beta_{0}, \beta_{2}$, and $\beta_{4}$ for the $\mathrm{K} 4 p_{3 / 2}-6 p_{3 / 2}$ system as a function of two-photon detuning $\delta$ for the three different dressing laser intensities $I_{d}=100,200$, and $500 \mathrm{MW} / \mathrm{cm}^{2}$. All other parameters are kept the same as those for Fig. 6, i.e., $\tau_{p}=1 \mathrm{~ns}, \tau_{d}=10 \mathrm{~ns}$, and $I_{p}=1 \mathrm{MW} / \mathrm{cm}^{2}$.

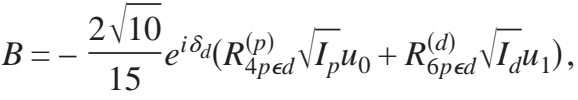

$$
\begin{aligned}
& C=\frac{\sqrt{2}}{3} e^{i \delta_{s}} R_{6 p \epsilon s}^{(p)} \sqrt{I_{p}} u_{1}, \\
& D=-\frac{2 \sqrt{10}}{15} e^{i \delta_{d}} R_{6 p \epsilon d}^{(p)} \sqrt{I_{p}} u_{1} .
\end{aligned}
$$

The variation of $\beta_{0}, \beta_{2} / \beta_{0}$, and $\beta_{4} / \beta_{0}$ is plotted in Fig. 8 . The highest-order coefficient $\beta_{4}$ is found to vary rather significantly, and we attribute this variation as the main reason for the striking variation of the 3D PAD we saw in Fig. 7.

\section{SUMMARY}

In summary, we have studied the modification of the photoelectron angular distribution by the introduction of a dressing laser. The role of the dressing laser is to induce so-called laser-induced continuum structure, thereby altering the branching ratio into different ionization channels. Since the partial photoelectron angular distribution is different for different continua, we expect alteration of the photoelectron angular distribution by the introduction of the dressing laser.

Specific numerical results are presented for the $\mathrm{K}$ $4 p_{1 / 2}-6 p_{1 / 2}$ and $4 p_{3 / 2}-6 p_{3 / 2}$ systems. Although it turned out that the photoelectron angular distributions are quite different for both systems, the alteration of the photoelectron angular distribution is striking, as we expect. Special care has to be taken, however, to avoid the undesired dynamic detuning originating from the ac Stark shift, since the dynamic detuning during the pulse can smear out the effects. For that reason, it is very desirable, as we have demonstrated in this paper, that the pulse duration of the probe laser is shorter than that of the dressing laser so that atoms experience practically static ac Stark shifts due to the dressing laser during the probe pulse. 


\section{ACKNOWLEDGMENTS}

G.B. acknowledges hospitality from the Institute of Advanced Energy, Kyoto University, during her stay, where the main part of this work was carried out. This work was supported by a grant-in-aid for scientific research from the Ministry of Education and Science of Japan.
[1] Y. L. Shao, D. Charalambidis, C. Fotakis, Jian Zhang, and P. Lambropoulos, Phys. Rev. Lett. 67, 3669 (1991).

[2] S. Cavalieri, F. S. Pavone, and M. Matera, Phys. Rev. Lett. 67, 3673 (1991).

[3] P. L. Knight, M. A. Lauder, and B. J. Dalton, Phys. Rep. 190, 1 (1991).

[4] Bo-nian Dai and P. Lambropoulos, Phys. Rev. A 36, 5205 (1987); 39, 3704 (1989).

[5] S. Cavalieri, M. Matera, F. S. Pavone, J. Zhang, P. Lambropoulos, and T. Nakajima, Phys. Rev. A 47, 4219 (1993).

[6] L. P. Yatsenko, T. Halfmann, B. W. Shore, and K. Bergmann, Phys. Rev. A 59, 2926 (1999).

[7] R. Eramo and S. Cavalieri, Opt. Commun. 149, 296 (1998).

[8] T. Halfmann, L. P. Yatsenko, M. Shapiro, B. W. Shore, and K. Bergmann, Phys. Rev. A 58, R46 (1998).

[9] K. Böhmer, T. Halfmann, L. P. Yatsenko, D. Charalambidis, A. Horsmans, and K. Bergmann, Phys. Rev. A 66, 013406
(2002).

[10] Takashi Nakajima and L. A. A. Nikolopoulos, Phys. Rev. A 68, 013413 (2003).

[11] Z. Chen, M. Shapiro, and P. Brumer, Chem. Phys. Lett. 228, 289 (1994).

[12] A. Shnitman, I. Sofer, I. Golub, A. Yogev, M. Shapiro, Z. Chen, and P. Brumer, Phys. Rev. Lett. 76, 2886 (1996).

[13] O. Faucher, E. Hertz, B. Lavorel, D. Charalambidis, R. Chaux, and H. Berger, J. Phys. B 32, 4485 (1999).

[14] O. Faucher, D. Charalambidis, C. Fotakis, J. Zhang, and P. Lambropoulos, Phys. Rev. Lett. 70, 3004 (1993); O. Faucher, Y. L. Shao, and D. Charalambidis, J. Phys. B 26, L309 (1993); O. Faucher, Y. L. Shao, D. Charalambidis, and C. Fotakis, Phys. Rev. A 50, 641 (1994).

[15] P. Lambropoulos and P. Zoller, Phys. Rev. A 24, 379 (1981).

[16] Takashi Nakajima, Jian Zhang, and P. Lambropoulos, J. Phys. B 30, 1077 (1997). 